Aus der histologischen Abteilung des pathologisch-anatomischen Institutes der böhmischen Universität in Prag (Vorstand Hofrat Prof. Dr. J. Hlava) und aus der venerischen Abteilung des k. u. k. Garnisonsspitales Nr. II in Prag (Kommandant Oberstabsarzt Dr. J. Schwarz).

\title{
Über den Verlauf und die histologischen Untersuchungen der luetischen Hyperkeratosen bei Behandlung mit Salvarsan.
}

\author{
Von \\ Regimentsarzt Dr. J. Odstrčil.
}

(Hiezu Taf. V.)

Lewin war der erste, welcher im Jahre 1893 die Clavi syphilitici histologisch untersuchte und den sich ergebenden Befund beschrieb.

Seit der Zeit wurde dieser syphilitischen Erscheinung keine besondere Aufmerksamkeit geschenkt, obzwar man sich nach Feststellung der Spirochaeten dem Studium der verschiedenen Effloreszenzen der allgemeinen Syphilis behufs Erforschung ihres Erregers fleißig gewidmet hatte. Eine Seltenheit bilden auch solche Arbeiten (Siegmund, Fischl u. a.), welche, wenn auch nicht neue Heilmittel, so doch wenigstens verschiedene Hg-Präparate zum Gegenstande haben, die bei Behandlung dieser hartnäckigen Syphilisform erprobt wurden. Deshalb faßte ich den Gedanken, diese Effloreszenzen zu studieren, um einesteils festzustellen, ob sie direkt durch Spirochaeten bedingt sind, anderteils ob wir im Salvarsan ein solches Heilmittel besitzen, welches den mit Clavi syphilitici Behafteten ron dieser sichtbaren Krankheit, die sich auch vor der Umgebung des Kranken nur schwer verheimlichen läßt, rasch befreit. 
Ich glaube auch in meiner Arbeit zu bestimmteren Resultaten dadurch gelangt zu sein, weil mir bei weitem mehr und bessere Färbemethoden zu Gebote standen, als es vor Jahren bei Lewin der Fall war, was auch einer von den Gründen sein dürfte, warum meine histologischen Ergebnisse mit jenen von Lewin beobachteten nicht vollkommen übereinstimmen.

Da Lewins Arbeit gewiß schon etwas in Vergessenheit geraten sein mag, erlaubte ich mir, in der Einleitung meiner Abhandlung, aus Lewins Publikation einen etwas umfangreicheren Auszug einzufügen, was mir hoffentlich nicht übel gedeutet werden wird.

Das großpapulöse Syphilid der Handflächen und Fußsohlen, schlechtweg Psoriasis syphilitica palmaris et plantaris genannt, zeichnet sich für gewöhnlich durch Bildung eines geringen, streng begrenzten Infiltrates und durch eine starke Schuppung aus. Das Infiltrat erscheint meist als ganz flach, in manchen Fällen jedoch hebt es sich an der Peripherie von der Umgebung schärfer ab und ist dann deutlich fühlbar. Manchmal kommt es jedoch nicht zu einer Abschuppung, sondern zu einer Hyperkeratose; es bäufen sich dicke, schwer ablösbare Hornhautauflagerungen an. An der harten, unnachgiebigen Haut entstehen dann in manchen Fällen an den Beugestellen und in den natülichen Falten Risse und es bilden sich schmerzhafte, oft tiefe Rhagaden. Die Erscheinungsweise dieser Syphilisform macht sich im allgemeinen in scharfbegrenzten, runden oder länglichovalen Infiltraten geltend; wenn aber benachbarte, krankhaft veränderte Stellen konfluieren, entstehen bogenförmig begrenzte, größere Infiltrate mit Hornmassenbildung, sogenannte "Clavi syphilitici".

Für die Diagnose ist der Umstand maßgebend, daß solche Erkrankungsherde sich immer nur auf die Volarfläche der Hände, der Finger oder auf die Plantarfläche der Fußsohlen beschränken. Von hier aus können die Klavi auch auf die Seitenflächen übergreifen, dabei zeigen sie aber stets eine bogenförmige Begrenzung; die Dorsalflächen bleiben jedoch verschont. Die Psoriasis syphilitica pflegt nach Mračeks Erfahrungen gewöhnlich erst 4-6 Monate nach der Infektion. 
oder noch später aufzutreten, mitunter auch oft nach vielen Jahren als ein spätes singuläres Rezidiv. Solche Fälle zeichnen sich dann durch einen schweren Verlauf aus und sind von langer Dauer, da sich nicht nur tiefere Infiltrate bilden, sondern auch größere Flächen der Handteller und der Fußsohlen davon ergriffen werden. Diese massigen, umschriebenen Hornhautwucherungen nennen wir, nach Lewin, wie schon früher erwähnt wurde, Clavi syphilitici; bei Entstehung hornähnlicher Gebilde sprechen wir von Psoriasis cornea oder Cornea cutanea luetica. Trotz ihrer Härte besitzen diese Hornmassenauflagerungen nicht die Dauerhaftigkeit der Clavi cutanei und werden entweder auf mechanischem Wege oder erst durch Ausheilung des syphilitischen Prozesses abgestoßen.

Lew in beschreibt die Clavi syphilitici als den gewöhnlichen Klavi ähnliche Gebilde, welche auf den Händen und den Füßen vorkommen, über das Hautniveau prominieren, hornartig beschaffen, hie und da von einem dünnen Schuppenkranz umgeben oder mit Schuppen bedeckt sind. Die Gebilde weisen die Größe eines Stecknadelkopfes bis einer Linse auf, sind kreisrund, oval oder von länglicher Form, bloß in zwei Fällen beobachtete Lewin eine größere Dimension dieser Gebilde. Sie liegen in der Haut wie eingekeilt, prominieren mebr oder weniger, allmählich nach oben sich verschmälernd; ihre Oberfläche ist meistenteils mehr plan, ja bisweilen sogar konkar, was Lewin in differential-diagnostischer Beziehung für wichtig bält.

Die Handflächen sollen öfters als die Fußsohlen betroffen werden. Stellen, wo die gewöhnlichen Klavi vorkommen, zeigen keine Prädilektion. Die Verteilung und das Auftreten der Clavi syphilitici folgt nicht dem Verlaufe der Hautnerven, auch nicht der Spaltrichtung der Haut, ebenso sind die Gelenkfalten der Handflächen und der Fußsohlen ohne Einfluß auf deren Lagerung; sie entstehen als blaßrote, erythematöse Flecke, die nach einer Zeit braunrot durch die Epidermis durchschimmern. Nach und nach rerdickt sich die über ihnen gelegene Hornhaut, welche sich später entweder in dünnen Schüppchen oder in größeren Lamellen ablöst oder sie verhärtet und es entstehen Klavi ähnliche Gebilde. 
Kranke mit syphilitischen Klavi behaftet, klagen entweder über geringes Jucken oder Brennen der Haut oder sie zeigen gar keine subjektiven Symptome. Die bei gewöhnlichen Klavi manchmal auftretenden, ausstrahlenden Schmerzen werden jedoch bei Clavi syphilitici nie konstatiert; auch Bewegungsstörungen werden nicht beobachtet.

Bei der Differenzialdiagnose kommen hier vor allem die Warzen, Hühneraugen, Schwielen und die Schuppentlechte in Betracht. Die Warzen prominieren bedeutend über ihre Umgebung, zeigen eine zerklüftete Oberfläche und finden sich oft gehäuft auf den Dorsalflächen vor. Für die Entwicklung der IIühneraugen bilden bekanntlich die prominenten Punkte an den Füßen die Prädilektionsstellen. Nach Kaposi lokalisieren sie sich aber in vereinzelten Fällen auch auf der Hohlhand; sie überragen die Umgebung und haben einen charakteristischen, zentralen, konischen Hornzapfen. Diagnostisch ist für das Hühnerauge der spontane stechende Schmerz und die hiedurch bedingte Behinderung des Gehens hervorzuheben. Den syphilitischen Klavi gleichen auffallend die Schwielen, was Farbe und Härte anbelangt, jedoch sind die letzteren in ihrer Form nie so scharf begrenzt und so kegelförmig wie die ersteren. Schwierig ist dio Diagnose, wenn sich die Psoriasis vulgaris, was man manchmal beobachten kann, ausschließlich auf der Hohlhand oder auf der Fußsohle lokalisiert; in diesen zweifelhaften Fällen empfiehlt sich heutzutage die Vornahme der serologischen Blutuntersuchung behufs Feststellung der richtigen Diagnose.

Es wäre nun noch $\mathrm{zu}$ erwähnen das Keratoma palmare et plantare und die Keratodermia symmetrica erythematosa (Besnier), bei denen einerseits das klinische Bild beider Erkrankungen, anderseits jedoch die auf der Haut auftretenden Erscheinungen oder anamnestische Daten für die Beurteilung maßgebend sind. Ernstere Erwägung verdienen noch jene Schwielenbildungen, welche als symptomatische Erscheinungen anderer Krankheiten, die auch isoliert auf den Hand- und Fußsohlen vorkommen, aufzutreten pflegen (Ekzem, Lichen ruber, Pityriasis rubra pilaris, Lepra); man muB aber auch darauf bedacht sein, daß durch toxische oder chemische länger- 
dauernde Reize, dann durch Hyper- oder Anhydrose, ja selbst durch Hysterie Schwielenbildungen hervorgerufen werden können. Nebst den aufgezählten Erscheinungen kommen auch noch im Verlaufe der Gonorrhoe Hyperkeratosen der Handteller und der Fußsohle vor und nach Angaben Buschkes wurden von Vidal, Jeanselm und $\mathrm{Ch}$ auffard solche beobachtet; ebenso müssen hier die Tylosis palmarum der Erwachsenen ( $\mathrm{Schüt}$ ) mit in Betracht gezogen werden.

Ob das eine oder das andere Geschlecht mehr zur Entwicklung der Clavi syphilitici prädisponiert ist, kann man in Anbetracht der geringen Anzahl der von Lewin beobachteten Fälle nicht entscheiden; unter seinen 28 Kranken waren 14 Männer und 14 Frauen. Nach dem Stande und der Beschäftigung der Mehrzahl seiner Kranken kommen mechanische oder traumatische Ursachen als auslösende Faktoren nicht in Betracht. Bei 7 Männern könnte wohl ihre Beschäftigung als Handarbeiter die Entstehung der Klavi begünstigt haben, doch gaben alle übereinstimmend an, daß sich die Klavi bei ihnen erst nach der syphilitischen Infektion entwickelten. Bei 5 Kranken saßen die Klavi auf der linken Hohlhand, obwohl sie nicht Linkshändler waren. Die Clavi syphilitici findet man am häufigsten bei Leuten, welche zwischen dem $20-30$. Lebensjahre stehen. Die Heredität hat keinen Einfluß auf ihre Entstehung, auch die Körperbeschaffenheit ist hier nicht von großer Bedeutung.

Die Clavi syphilitici können sich gleich im Anschlusse an die Sklerose ausbilden, sie stellen sich aber häufiger bei Rezidiven ein; treten auf vor und nach durchgemachten antiluetischen Kuren, am häufigsten kombiniert mit makulösen und papulösen Exanthemen; bei späteren sekundären Symptomen sind sie seltener, am seltensten aber bekommt man sie in dem tertiären Stadium zu Gesicht. Die Kiari pflegen häufig fast gleichzeitig beide Handteller und Fußsoblen zu befallen, welcher Umstand schon daraut hinweist, daß sie nicht durch lokale Einflüsse erzeugt werden, sondern daß sie die Folge einer Allgemeinerkrankung sind.

Wie die Statistik Lewins nachweist, handelte es sich in $38 \%$ der Fälle nur um einseitige Lokalisation des Klavus, 
welcher Umstand gewiß diagnostische Schwierigkeiten bereiten kann. Lew in behandelte seine Kranken mit täglichen subkutanen Sublimatinjektionen à $0.1-0.12 \mathrm{~g}$. Bei 23 Kranken machte er ca. 386 Injektionen, was durchschnittlich ca. 17 Injektionen bei einem Falle entspricht; die Heilung der Klavi wurde durch Einverleibung von ca. 0.204 $g$ Sublimat erzielt.

Schon den Syphilidologen des 15. Jahrhundertes waren luetische Erkrankungen der Handflächen und Fußsohlen bekannt. Erst die Autoren der letzten Dezennien des 18. Jahrhunderts weisen darauf hin, daß die Psoriasis palmaris et plantaris selbständig ohne gleichzeitige Komplikation mit anderen syphilitischen Erscheinungen auftreten kann. Nach einer langen Pause, d. i. in den vierziger Jahren des vorigen Jahrhunderts, wird namentlich von französischen Autoren eine besondere Form als Psoriasis cornée beschrieben.

Fournier und Cohn haben unter der Bezeichnung Syphilide cornée und Psoriasis palmaris cornea Effloreszenzen beschrieben, welche den von $L$ ewin beschriebenen am meisten ähnlich sind. Kaposi, v. Zeissl, Hutchinson, Bumstead, Taylor, Lesser, Reder, Behrend, Hill, Campana, During, M. v. Zeissl und Finger geben in ihren Lehrbüchern und Abhandlungen eine mit den oben angeführten Symptomen sich deckende Beschreibung der syphilitischen Klavi an.

Als ich heuer, bei Beginn der neuen Behandlung mit Salvarsan, einen Kranken mit sogenannten Clavi syphilitici meinem ehemaligen Lehrer P. T. Prof. Janorský vorführte, gab er mir den Rat, nicht nur die Veränderungen an Ort und Stelle, die diese Krankheitsformen unter dem Einflusse des Arsenobenzols erleiden, eingehend zu beobachten, sondern auch einige "Clavi syphilitici" vor und während der Behandlung zu exzidieren, auf Spirochaeten zu färben und die Schnitte dann histologisch unter Anwendung verschiedener Färbemethoden zu untersuchen. Dem Rate folgte ich gerne, da ich im ganzen bloß 4 Fälle von Clavi syphilitici unter 1500 Syphilitikern zu beobachten Gelegenheit hatte.

I. F all. Anfangs Oktober $1910 \mathrm{kam}$ in die Behandlung des Garnisonsspitals Nr. 11 der Rekrut J. G., 21 Jahre alt, mittelgroß, schwächlich, von sehr blassem Aussehen, die sichtbaren Schleimhäute leicht anä- 
misch, von $59 \mathrm{~kg}$ Körpergewicht. In der Kindheit machte derselbe Masern, später Mumps durch, sonst war er nie krank gewesen. Patient ist der jüngste unter 9 Geschwistern. Seit 16 Monaten war der Kranke als Heizer auf einem Kettendampfer angestellt. Den 3. April 1910 war der erste und zugleich der infektiöse Koitus. Ende Juni bemerkte der Kranke, daß sich die Vorhaut des Gliedes blaurot verfärbt und sich nicht mehr wie früher zurückziehen läßt und daß das Glied selbst an Stärke zunimmt. Zur selben Zeit bildete sich an mehreren Stellen beider Handteller und Fufsohlen, nach seiner Angabe, eine Schwiele, die er mit dem Worte "Huf" bezeichnete. Der Kranke gab an, daB er zuerst in der Mitte der rechten Hohlhand eine begrenzte linsen- bis hellergroße Rötung vemerkte, über welcher sich bald eine harte Haut ansanmelte; später war es die andere Palma und die Finger. Nach einigen Tagen beobachtete er zu seiner Überraschung, daß auch an beiden Fußsohlen äbnliche Gebilde hervortraten, was ihn jedoch bei seiner Arbeit und bei dem langen Stehen nicht behinderte und auch nicht schmerzte. Im Monate August zeigte sich noch ein Ausschlag auf dem Körper; seit 3 Wochen bemerkte er ein Wundsein und Nässen um den After und zwischen den einzelnen Zehen beider Füße.

Status praesens. Organbefund normal, Augenspiegelbefund desgleichen; die Harnproben ergeben nur eine leicht positive Diazoreaktion, Urobilinprobe negativ. Die sichtbaren Schleimbäute bla $\mathfrak{B}$, die Haut des Körpers gelblichbraun, im Gesichte und auf den oberen Rückenpartien zahlreiche Aknepusteln und Komedonen, dazwischen oberflächliche, kleine, eingesunkene Narben. Das Haar vorne etwas schütter. Die Haut des Halses und Nackens braunverfärbt mit 22, teils vereinzelten, teils gruppierten linsen- bis hellergroßen, rundlichen oder verschieden geformten Leukodermaflecken besät. Die Halsdrüsen erbsengroß, die linke Kubitaldrüse kaffeebohnengroß, die rechte reiskorngroß. In der rechten Leistengegend 4 , in der linken 5 vereinzelte bohnengroße, derbe, indolente Drüsen. In dem oberen Segmente beider Brustwarzenhöfe finden wir je eine Effloreszenz eines etwa hellerstückgroßen, orbikulären, kleinpapulösen Syphilids. Am ganzen Körper, besonders aber an den Streckseiten beider unteren Extremitäten, dann in der Kreuzbein- und Gesäßgegend sehen wir ein, meist in unregelmäßigen Gruppen auftretendes lichenoides Exanthem. Die zahlreichen kleinen syphilitischen Knötchen glänzen stark und tragen besonders in der Schienbeingegend schmutziggraue, festhaftende Schüppchen. Der Nabelring ist stark infiltriert, hervortretend; der Nabeltrichter mit vielen dicht aneinander gereihten, braunroten, miliaren Papeln besetzt. In den oberflächlichen Falten bemerken wir eine ausgiebige Schuppung, in den tieferliegenden Falten und auf den Kontaktflächen dagegen starkes Nässen. Der Penis ist durch ein sklerotisches Ödem auf das doppelte verdickt; die ganze Vorhaut ist stark entzündlich infiltriert, besonders am Rande; nicht reponibel, blaurot verfärbt; auf deren Oberfläche glänzende, trockene, miliare bis linsengroße, dicht gesäte Papeln. An der Haut des Hodensackes ähnliche Effloreszenzen. 
Um das Orificium urethrae eine zirkuläre zweihellerstückgroße, braunrote, hypertrophische Papel, links und nach innen davon an der Glans zwei linsengroße, nässende Papeln. In den Analfalten und zwischen den Nates drusige, himbeerartige, einige Millimeter hohe, graubelegte, stark sezernierende, breite Kondylome, welche die Analöffnung völlig verdecken. Beide Mandeln stark vergrößert, ihr Epithel milchig getrübt; an dem oberen Pole der linken Tonsille ein bellergrobes, vertieftes, speckig belegtes Geschwür. In der Hohlhand beiderseits, sowie auf den Volar- und Kontaktflächen sämtlicher Finger sind im ganzen rechts 21, links 23, teils vereinzelte, teils gruppierte zu 2-3, linsen- bis hellergroße, runde oder ovale, scharf begrenzte, schwielenähnliche Stellen, von denen meisten mit einer derben, sehr dicken Hornschicht bedeckt sind. Die Stärke der Hornschicht ist über allen betroffenen Stellen keine gleichmäßige. Die Oberfläche ist entweder intakt, oder es zeigen sich parallel mit den $n_{\diamond}^{\circ}$ Beugefurchen verlaufende oder dieselben kreuzende, verschieden tiefe und breite Rhagaden, so besonders an den vorspringenden Ballen. Die Hornmassen haben eine geibliche, leicht durchscheinende Farbe, oder, wenn in die Rhagaden Verunreinigungen eindringen, einen mehr schmutziggelben Ton. Diese Massen halten fest zusammen, sind derb oder mitunter wenig nachgiebig; in der Hohlhand beiderseits zeigen sie sogar eine gewisse Brüchigkeit. An einzelnen Stellen hat sich die brüchige Hornschicht abgestoßen und nur einen ausgefransten Rand zurückgelassen mit einer vertieften Basis und einer mäBig infiltrierten Peripherie.

An der linken Fußsohle, besonders an der unteren Fläche des ganzen äußeren Randes und der Ferse, dann in der Gegend sämtlicher Zehenballen befinden sich 23 mit dicken, gehäuften Hornmassen bedeckte, gelblichweiße, runde oder längliche Clavi syphilitici in der Größe einer Linse bis eines Kronenstückes, teils vereinzelt, teils zu 2-3 gruppiert. An dem inneren Rande des Fußgewölbes 4 braunrote, ca. hellergroße, schuppende Papeln. In vielen mit der spröden Hornschicht versehenen Klavi findet man seichte Einrisse bauptsächlich auf beiden Fubsohlen. Derselbe Befund (11 Klavi) an der rechten Fußsoh]e. Die Sensibilität der betroffenen Stellen ist sowohl für taktile, wie auch für thermische Reize erhalten, jedoch ist die Perzeption verlangsamt. Die Beweglichkeit der Extremitäten ist in keiner Weise eingeschränkt. Die betreffenden krankhaften Teile sind schmerzlos und ohne unangenehme Parästhesien. Die Affektion ist prägnant dort entwickelt, wo die Sohle für gewöhnlich einem starken Drucke ausgesetzt ist, so auf der Plantarfläche der Ferse, dann plantar und median am Groß- und Kleinzellenballen, jedoch auch in der Medianlinie der Soble finden sich einige kleinere Schwielen vor. An den Füßen und Händen ist eine stärkere Schweißsekretion bemerkbar. $Z$ wischen der vierten und fünften $Z$ ehe rechts und der dritten und vierten links hochgradig mazerierte Papeln.

Spirochaeten wurden im Reizserum der nässenden Papeln an verschiedenen Stellen, gefärbt nach Giemsa und Burri, nachgewiesen. Die Wassermannsche Seroreaktion war stark positiv. Tags vor der 
Injektion wurden einzelne kleine Klavi ganz, aus den größeren kleinere Partien ausgeschnitten und in Formol gehärtet.

Den 12. Oktober vormittags: Injektion mit $0.5 \mathrm{~g}, \mathrm{Ehrlich}-\mathrm{Hat} a$ 606", hergestellt nach Blas chko; Einspritzung in der Menge von $10 \mathrm{~cm}^{3}$ rechts intragluteal in drei von einanàer getrennte Stellen. Höchste Temperatur 10 Stunden nach der Injektion um $9 \mathrm{Uhr}$ abends $37.5^{\circ}$, bis inklusive 18. Oktober geringere Temperatursteigerungen, lytischer Abfall; von da ab immer normale Körperwärme. Erst den 15. Oktober bemerkten wir, daß in der Hohlhand beiderseits deutliche Schuppung eingetreten ist, einzelne vertiufte Narben gleichen sich aus; die harten Hornmassen an beiden Fußsohlen werden stellenweise nachgiebiger. Den 16. Okt. lösen sich in beiden Hohlhänden an einzelnen Stellen die Hornmassen in kleinen, trockenen Lamellen ab. An den Fußsohlen sind die Klavi weich, nachgiebig, doch die Hornmassen in früherer Stärke noch vorhanden. Den 17. Okt. sind die schuppenden Papeln in der Fulßhöhlung mit Hinterlassung braunroter Pigmentationen abgeheilt. Den 18. Oktober zeigen drei nebeneinander stehende Klavi in der Mitte der rechten Hohlhand einen kompletten Abfall der Hornmassen und es wird bereits eine frische, rosarote, mit normaler Hautzeichnung versehene Epidermis sichtbar; links schreitet die Abschuppung fast noch rascher vorwärts. Den 21. Okt. sind die Hornmassen an beiden Fußsohlen stark ausgetrocknet und weisen tiefe Rhagaden und an einzelnen Stellen sich ablösende kleine, dicke, geschichtete Lamellen auf.

Den 22. Oktober heben sich die Hornmassen in dicken, aber schon größeren Lamellen ab. In der Hohlband beiderseits sind die Hornmassen fast überall in dünnen Schuppen abgefallen und an ihre Stelle tritt dort eine rosarote, normale, leichtvertiefte Epidermis. Den 24. Okt. lösen sich an beiden Fußsohlen die aufgelagerten Hornmassen in ausgiebigem Maß3e in großen, breiten Lamellen ab. Den 25. Okt. sind an beiden Händen die erkrankten Stellen abgeheilt und es ist keine Abschuppung mehr vorhanden. Den 30. Okt. Beide Fußsohlen von normaler Beschaffenheit, Farbe und Konsistenz. Der Kranke durfte während der ganzen Beobachtungszeit weder ein Hand- noch Fubbad nehmen; lokal indifferente Behandlung. Auch wurde derselbe von anderen mit der Schmierkur behandelten Kranken getrennt gehalten.

II. F a ll. Ende Dezember 1910 kam ein kranker N. N. mit sog. Clavi syphilitici ins Garnisonsspital in Behandlung. Dieser Fall ist um so interessanter, als der Kranke bereits vor 4 Jahren Lueserscheinungen an denselben Stellen hatte, wo sie auch diesmal zu sehen sind. Weil damals unter $\mathrm{Hg}$ - und Jodkalibehandlung die Effloreszenzen nur langsam schwanden, also hartnäckig wie gewöhnlich waren, sprach der Kranke den Wunsch aus, diesmal mit Salvarsaninjektion behandelt zu werden, welches Ersuchen ich mit Rücksicht auf den in dem ersten Falle erzielten günstigen Erfolge, erfüllte.

Anamnestisch ist aus seiner Kindheit nichts Besonderes zu verzeichnen. Vor 10 Jahren akute Gonorrhoe. Im J. 1905 Ulcus durum mit 
einem makulösem Exanthem. 6 Einreibungstouren. Ein Jahr darauf trat ein papulöses Syphilid am Körper und Plaques muqueuses auf den Schleimhäuten auf; zugleich war an beiden Hand. und Fußflächen eine Psoriasis palmaris et plantaris 20 sehen. Eine ausgiebige $\mathrm{Hg}$-Kur mit nachfolgenden Jodkalidosen brachten erst nach zwei Monaten an den letztgenannten Stellen den Prozeß zur Abheilung. Erst den 17. Dezember 1910 machte sich die Lues von Neuem durch ein Hand- und Fußflächensyphilid bemerkbar.

Status praesens. Patient mittelgroß, kräftig gebaut, gut genährt. Die inneren Organe, ebenso der Augen- und Augenspiegelbefund normal. Nervenstatus ohne pathologische Erscheinungen. Im Harne kein Eiweiß, kein Zucker, kein Urobilin.

Die Haut der Handteller und Fußsohlen anffallend trocken; nebst der normalen Felderung bemerkt man noch verschieden sich kreuzende Furchen, so besonders am Thenar und Hypthenar. Diese Stellen zeigen eine ausgiebige, kleienförmige Abschuppung. In der Mitte der rechten Hohlhand sehen wir eine zweihellerstückgroße, braunrote, mäßig vorgewölbte, beim Kratzen leicht schuppende aber nicht blutende Papel. In der linken Hohlhand sind fünf bis einhellerstückgroße, braunrote, etwas vertiefte, mit einem ausgefransten Rande versehene Papeln, über welchen die Hornhaut geschwunden ist; drei ähnliche, unregelmäßig gestaltete Papeln findet man an der Beugeseite des linken Handgelenkes radialwärts, in einer schräg von obeu innen nach außen unten verlaufenden Linie.

Die Haut der Fußsohlen, der Fersengegend und des äußeren Fußrandes in der Breite von $2 \mathrm{~cm}$ zeigt die gleiche trockene, stark gefelderte und schuppende Beschaffenheit, wie oben an den Händen geschildert. Die Haut direkt über beiden Knöcheln, dann hauptsächlich am ganzen äußeren Fußrande beiderseits, von der kleinen Zehe um die Ferse bis zum inneren Knöchel, ist in der Breite von $2 \mathrm{~cm}$ mäßig infiltriert und mit teils vereinzelten, teils gruppierten (zu 3-4 an den Knöcheln), linsengroßen, braunroten, mit harter Hornschicht versehenen, mäßig erhabenen Papeln besät. An der Kuppe der linken Fußhöhlung nahe der Mittellinie der Fußsohle findet man drei stecknadelkopf- bis linsengroße, von einander getrennte, braunrote, mit harter, hoher Hornsehicht versehene Gebilde, sogenannte "Clavi syphilitici". Die Hornmassen sind gelbbraun, die Oberfläche rauh aber intakt. Eine Vornahme der Exzision der Klavi wird vom Kranken hartnäckig verweigert.

Am rechten Brustbeinrande, in der Höhe der dritten Rippe, eine zweihellerstückgroße, braunrote, schuppende, elevierte Papel. Den 27. Dez. 1910 wurde 0.6 Salvarsan in $8 \mathrm{~cm}^{3}$ (Suspension nach B la sch ko) intragluteal, links außen, an 4 verschiedenen Stellen, aber im selben Einstichkanal, deponiert. Die höchste Temperatur den 29. Dez. um 6 Uhr abends $37 \cdot 1^{\circ}$ C. Den 31. Dez. ist die Abschuppung an allen Stellen minimal, die elevierten Papeln abgeflacht, die Randinfiltrate im Niveau der Umgebung, die ausgefransten Rändersäume sind nicht mehr zu sehen. Über den „Clavi syphilitici" hat sich die harte, dicke Hornschichte in trockenen, 
spröden Lamellen abgestoßen; an deren Stelle ist eine rosarote, mäßig vertiefte Delle mit normaler Zeichnung und nur eine schmale, etwas infiltrierte Randzone zu sehen. An Stelle der Papel am Sternum eine braungelbe Pigmentnarbe. Den 3. Januar 1911 erscheinen die restlichen Infiltrate gänzlich resorbiert mit Hinterlassung braungelblicher Pigmentnarben.

III. F a ll. Auch wollte es der Zufall, daß noch ein dritter Kranke auch mit „Clavi syphilitici“ behaftet in Behandlung unseres Spitals gekommen ist.

Aus der Anamnese sind nur Scharlach und Diphtherie im Kindesalter hervorzuheben. Geschlechtlich früher nie krank gewesen. Die Ansteckung erfolgte anfangs Dezember 1910, die Sklerose wurde mit grauem Pflaster behandelt. Am 27. Januar bemerkte der Kranke im Gesicht, auf der Brust und im bebaarten Kopfteile einen Ausschlag. Bei seiner Aufnahme ins Garnisousspital am 31. Januar folgender status praesens :

Patient mittelgroß, sehr kräftig, stark fettleibig. Im Gesichte, besonders auf der Stirne, dann in der linken Nasolabialfalte und in der Kinnfalte gehäufte, meist linsengroße, elevierte, braunrot verfärbte Papeln, in beiden seitlichen Thoraxpartien und am Rücken einzelne zerstreute papulöse Effloreszenzen. Im behaarten Kopfteile dichtgesäte Papeln vermengt mit Pusteln. Beide Tonsillen gerötet, stark vergrölßert, mit Plaques muqueuses. Auf der Oberlippe rechts von der Mittellinie eine zerfallene Papel. An der linken Hohlhand am Thenar und Hypothenar je ein reiskorngrober, mit sehr harter Hornschichte versehener, kegelförmiger, rotbraun verfärbter Klavus, desgleichen waren auf der inneren Kontaktfläche des rechten Goldfingers anf der zweiten Phalanx zwei von einander getrennte ähnliche Gebilde, sonst Hände und Fußsohlen frei. Im Saleus coronarius links von der Mittellinie eine leicht verbärtete, mäßig vertiefte rosarote Narbe. Anus, Genitale frei. Eine Exzision der Klavi wird verweigert. Organbefund normal, ebenso Nerven- und Augenspiegelbefund. Der Harn zeigt deutiche Urobilinreaktion, kein Zucker, kein Eiweiß.

Den 3. Februar Salvarsaninjektion intragluteal beiderseits zu je $5 \mathrm{~cm}^{3}$ (Suspension nach Blaschko). Höchste Temperatur $389^{\circ} \mathrm{C}$ am 2. Tag 6 Uhr abends.

Den 8. Februar nahmen die "Clavi syphilitici“ eine gelblichbraune Verfärbung an, sonst sind sie unverändert. Den 12. Februar verlieren sie ibren Glanz, ihre Konsistenz wird etwas nachgiebiger. Den 15. Februar zeigt der Klavus am linken Hypothenar mattweiße Farbe, und die Hornschicht feine Risse. Am 18. Febr. weisen auch die drei anderen Klavi dieselben Veränderungen auf. Den 20. Febr.: Über allen „Clavi syphilitici ${ }$ heben sich kleine Lamellen der Hornschichte ab; die Konsistenz der Klavi geringer. Den 24. Febr.: Die harte, abgehobene Hornschicht löst sich über den vier Klavi allmählich in dünnen Schüppchen ab, nachher sind kleine rosarote Dellen sichtbar.

Auch am übrigen Körper sind alle Effloreszenzen mit Hinterlassung braunroter Pigmentnarben abgeheilt. 
Von Bedeutung ist, daß diese harten Gebilde dem Kranken gar keine Beschwerden verursachten, denn er erfuhr ihre Existenz erst durch meine Untersuchung.

Eine mechanische Ursache bei der Entstehung der „Clavi syphilitici“ ist hier nicht anzunehmen, da der Kranke seit Jahren nur Kanzleidienste versah und auch in den letzten drei Monaten keinen Sport ausübte. Eine lokale Behandlung der Clavi wurde während der Spitalsbehandlung nicht vorgenommen.

\section{Histologischer Befund.}

Im Korium reicht eine Infiltration von kleinen, einkernigen Rundzellen fast bis auf das tiefe Gefäßnetz; dort, wo die Gefäßverzweigungen stärker sind, sehen wir mächtigere Infiltrate in Form verschieden breiter Zellstränge, oder bildet das kleinzellige Infiltrat knötchenförmige Nester um die kleinen Gefäße. Stellenweise durchdringt das Infiltrat auch die Gefäßwandungen, reizt hier das Endothel zur Wucherung und Verschmälerung des Gefäßlumens. Um die feinsten und ganz zarten Endverästelungen der Gefäße des Papillarkörpers und um die des Rete subpapillare fließen die einzelnen Infiltrate mit dem geschwellten Endothel zu breiten, dichten, streckenweise streifenförmigen, einheitlichen Infiltrationsherden zusammen. Diese fast die ganze Dicke des Koriums einnehmende Zellinfiltration, welche aus Lymphozyten mit schmalem, stellenweise nicht sichtbarem Protoplasma und aus reichlichen Plasmazellen besteht, dringt mit den papillaren Gefäßschlingen bis hoch in die Spitzen des Papillarkörpers und bildet hier überall einen dichten Herd. Die Anhäufung von Plasmazellen zeigt auf einen länger dauernden Prozel hin; nach Ehrmann besteht das Infiltrat in der ganz frischen Papel aus Leukozyten und erst später treten reichlich Plasmazellen auf.

Das aus der Tiefe kommende, an die Gefäße gebundene Infiltrat dringt an manchen Stellen bis in die basalen Schichten des Stratum Malpighii ein, ja wir finden selbst zwischen den einzelnen Retezellen in den interzellulären Lücken deformierte Leukozyten vor. (Mourek.)

In den Schnitten des 6 Tage später exzidierten Hautstückes (Klavus) finden wir in dem Stratum papillare und in 
der Subkutis eine teils zerstreute, teils die Gefäße begleitende kleinzellige, ziemlich dichte Infiltration, welche auch in die Papillen hinaufdringt. Doch ist dieselbe nicht mehr so gleichmäBig und so dicht; einzelne Papillen sind nur wenig ausgefüllt; dagegen sieht man in deren Nachbarschaft wieder solche, die eine dichtere, diffuse Anschoppung mit Infiltratzellen zeigen.

In beiden Reihen von Präparaten sehen wir auch, daß die Schwoißdruisen von einem Zellinfiltrat umsponnen sind.

Zwischen den Zellanhäufungen ist bemerkbar eine Wucherung der fixen Bindegewebszellen mit zahlreichen großen Spindelzellen, letztere mit einem blassen Kern und lang ausgezogenem Protoplasma. Auch erscheinen die Bindegewebsfibrillen und Bündel etwas dichter und dicker als im normalen Zustande; selbst in die Papillen steigen verdichtete, geschlängelte Bindegewebsfibrillen auf.

Die elastischen Fasern, die nach Se c chi normalerweise in der Haut der Vola manus in die Papillen in beträchtlicher Menge eindringen und an der Basis derselben und unter der Rundung der interpapillaren Retezapfen ein dichtes Netz von dünnen gestreckten und dickeren geschlängelten Fasern bilden, sind zum Teile zugrunde gegangen. Wir finden nämlich in unseren Präparaten, daß die Menge der elastischen Fasern gegen das Normale etwas spärlicher ist; an Stelle des subpapillaren, früher dichten Netzes sieht man jetzt mehr locker geflochtene Kuäuel; auch in die Papillen verlaufen nur einzelne dünne oder dickere geschlängelte Fäserchen, die frühere gleichmäBige Verteilung fehlt. Im Korium besteht das elastische Gewebe stellenweise aus dickeren Fasern, sonst aber sind die Verhältnisse normal.

Die Papillen sind nur an jenen Stellen vergrößert und verlängert, an welchen sich im Papillarkörper ein reichliches, dichtes Infiltrat angehäuft hat, anderswo weichen sie in ihrer Form und Größe nicht von dem gewöhnlichen Aussehen ab.

Die Basalschicht der Epidermis zeigt 1-2 Reihen zylindrischer Zellen, deren Protoplasma sowie die Form des Kernes und auch dessen Lagerung keine Besonderheiten aufweisen; diese Zellen stehen wie normal senkrecht auf der Kutisober- 
fläche. Diese Schichte ist regelmäBig angeordnet und ist gegen das Bindegewebe gut abgegrenzt. Eine Abrundung der Zylinderzellen an der Spitze sowie eine Faß-oder Eiform derselben (Mourek) konnten niemals beobachtet werden. Hie und da dringt das kleinzellige Infiltrat zwischen die Basalzellen.

Das Stratum spinosum umgibt die Papillen in ziemlich mächtiger Schichte. Die Zellen sind regelmäßig angeordnet; meist rhombischer Form, hie und da etwas in die Länge gezogen, ihre Kerne sind meist gut entwickelt und deutlich sichtbar und haben eine runde oder längliche Form. Die Gestalt und die Größe einzelner Zellen weicht von der Norm nur sehr wenig ab.

Die Interzellularbrücken resp. Stacheln sind gut entwickelt, ja es scheint, als wären sie etwas breiter.

Hie und da sieht man im Stratum spinosum Vakuolisation der Zellen.

Pigment auch in Form feinster Körnchen war weder in den tiefen Reihen der Basalzellen, noch auf den aufsteigenden Wänden der Papillen nachweisbar. Auch konnte man Pigment enthaltende Bindegewebszellen (Pigmentophoren) weder vereinzelt, noch in Gruppen unabhängig vom Verlaufe der Gefäße wahrnehmen.

Die Untersuchungen der Serienschnitte von beiden Präparaten (vor und nach der Injektion) ergaben bezüglich des Nachweises von Spirochaeten nur negative Resultate (Färbemethode nach Levaditi).

In allen Schnitten ist das Stratum granulosum in der ganzen Ausdehnung deutlich und mächtig in 5-7 Zellagen ausgebildet. In den obersten vier Reihen sind die Zellen mit Keratohyalinkörnern völlig vollgepfropft, so daß3 das Protoplasma kaum sichtbar ist. Nach Färbung mit Hämatoxylin bemerken wir direkt unter dem Stratum corneum eine breite, diffuse, blauschwarz gefärbte Zone, welche auffallend von der blaßgrauen Hornschicht absticht. Durch diese Anschoppung mit Keratohyalinkörnern sind die Kerne der Zellen in dem Stratum granulosum zum großen Teil bedeckt oder sogar ganz eingehüllt. Die Anhäufung des Keratohyalins an der Peripherie der Kerne läßt ihre Kontur scharf erscheinen und ihre Lage 
in den Zellen ist meist exzentrisch. Die Kerne selbst färben sich sehr schwach, ihre Kernkörperchen aber deutlich und diese sind gewöhnlich exzentrisch gelagert. In den tieferen Lagen des Stratum granulosum sind dagegen die Zellen mit feinkörnigem Keratohyalin gefiillt und heller gefärbt, hier liegen die Kerne meist zentral, ihre Konturen sind nicht so scharf angedeutet und die Kernkörperchen sind verschieden gelagert. Die Begrenzung der Zellen selbst ist mit Ausnahme der obersten Reihe scharf, deutlich sichtbar.

Die Peripherie der Zellen ist uiberall frei von Keratohyalin, man trifft auch nie in den Interzellularbrücken Keratohyalinkörner an. Unna und Dubreuilh meinen, daß bei Schwielen im Stratum granulosum mehr Zellen vorhanden sind, die Keratohyalin enthalten, daß aber dngegen jede dieser Zellen weniger als normal Körner enthalten. In unseren Schnitten aber finden wir nicht nur die Zellreihen, sondern auch das Keratohyalin vermehrt.

Die oberste Reihe des Stratum granulosum in unseren Schnitten zeigt Zellen, deren Konturen oft verwischt sind, so daß die Körner scheinbar frei liegen; an diesen Stellen und dann in dem basalen Teile der Hornschicht findet sich Ele id in in schmalen Schichten vor. Flache, kernhaltige Hornzellen, die man im normalen Zustande in dieser Höhe oft zu Gesicht bekommt, sind nirgends nachzuweisen. Die Zellen dieses Stratums sind mehr in die Breite gezogen, verändern durch die Hautspannuvg und den Druck von außen gegen die Oberfläche ihre Gestalt und werden nach oben zu immer platter.

Wesentliche Veränderungen weist die Epidermis auf; ihre Hornschicht zeigt nämlich eine außerordentliche Mächtigkeit, Vermehrung und Ausdehnung; das Hornzellenlager milst an Stellen der größten Fintwicklung ca. $0.6-0.8 \mathrm{~mm}$, die einzelnen Lamellen hängen fest zusammen und bilden eine dichte Schichte. Gegen das Stratum granulosum hin begrenzt sich diese Schichte scharf mit einer leicht welligen Linie, ohne daß man ein Stratum lucidum so deutlich wie im normalen Falle wahrnehmen könnte; nur hie und da ist eine Andeutung desselben vorhanden, nur an der Peripherie einzelner sehr dünnen Paraffinschnitte findet man eine schmale, homogene, glänzende Schichte 
zwischen dem Stratum granulosum und den aufgetürmten Hornzellschichten eingelagert, die man nicht ohne Grund als Stratum lucidum deuten könnte; diese letztgenannte Schichte verbält sich anders gegen verschiedene Farbstoffe als die übrige obere Hornschichte; sie färbt sich nach van $G$ i e s o n schwach gelb, aber mit Polychrommethylenblau gar nicht. Zellen aber oder Kernreste sind daselbst nicht färbbar. Diese basale Hornschichte, die der $\mathrm{Oehl}$ schen Schichte entspricht, zeigt in Gefrierschnitten ziemlich viele mit Kongorot färbbare schmale Stränge von Kernen, die wir als Eleidin bezeichnen.

Die Hornmassen bestehen aus Hornlamellen, die ein dichtes parallel übereinander liegendes Hornzellager bilden, einen leicht wellenförmigen Verlauf zeigen und meistens kontinuierlich zusammenhängen; nur stellenweise sind Spalten oder Lücken sichtbar. Diese Massen zeigen an keinem Orte färbbare Kerne.

Häufig ist in den Schnitten das Stratum corneum durchzogen von vielen Schweißdrüsenausführungsgängen von korkzieherartiger Form, um welche wohl eine starke Farbstoffansammlung zu sehen ist. Die Struktur der Wandungen der Ausführungsgänge ist verwischt.

Nach Unn a besteht die Hornschicht aus einer verdickten, verdichteten und kernlosen Endschicht und aus einer melr oder weniger breiten Mittelschicht, in der unsichtbare Kernreste mit Orzein-Fuchsin- und Kreosotenfärbung deutlich zutage treten. Dubreuilh und Sklarek behaupten aber, daß sie mit den oberwähnten Färbemethoden Unnas in dem Stratum corneum der unzweifelhaften Schwielen färbbare Kerne in größerer Zahl fast nie nachweisen konnten.

Die Hornzellen selbst sind nach Unna Oberhautzellen, welche makroskopisch ein trockenes, ziemlich hartes, verschieden transparentes Gewebe darstellen und bei Pepsinsalzsäurebehandlung das Keratin zurücklassen.

Was die Härte der Hornsubstanz betrifft, steht diese mit dem Keratingehalt in keinem proportionellen Zusammenhange. Aber auch an der Anhäufung der Hornmassen ist nach Unnas Verdauungsversuchen nicht der Keratingehalt der Einzelzellen 
schuld, sondern dieselbe wird bedingt durch Zusammenbalten der geschweißten Hornschichte.

Die Kohärenz der einzelnen Elemente in starke Hornschichten ist ermöglicht durch das Erhaltenbleiben eines rudimentären Stachelpanzers in Form zahlreicher Verbindungsbrücken. (Unna, Rausch, Weidenreich.)

Nach Ansicht Janovskýs und Neisser-Jadassohns kann bei essentiellen Hyperkeratosen die pathologische Verhornung durch Hyperplasie in der Malpighischen Schichte (mit oder ohne Akanthose) entweder 'durch direkten Reiz oder durch diskontinuierlichen Druck, welcher bald Anämie bald Hyperämie bewirkt (Epithel-Hornschicht-Hypertrophien, $\mathrm{N}$ e is s e rJadassohn), oder eventuell auch durch trophische Einfliusse eingeleitet werden. Alle diese Reizmomente können dann tiefer bis in die Papillarschichte iubermittelt werden.

Über das Wesen und den Beginn des Verhornungsprozesses herrschten bis jetzt keine einheitlichen Anschauungen.

Dem Stratum granulosum mit dessen Keratohyalinkörnern wird bei der Bildung der Hornschichte eine große Bedeutung und wichtige Rolle beigemessen; doch in welchem Grade dies mit Recht geschieht, wurde noch nicht festgestellt.

Nach A ufhammer, Waldeyer, Unna werden nicht das Keratohyalin, sondern auch das Eleidin als normaler Bestandteil der Epidermis angesehen und beide diese Substanzen pfleren bei dem normalen Verhornungsprozesse immer vorhanden zu sein. In welchem Verhältnisse diese beiden Substanzen zueinander stehen, ferner welche von ihnen die größere und wichtigere Rolle bei der Verhornung spielt und welche Bedeutung überhaupt man ihnen dabei zuschreiben darf, darüber differieren die Ansichten noch immer sehr stark. Fast alle Autoren stimmen aber darin überein, daß bei der Verhornung normalerweise beide Substanzen sich bilden; ob derselbe Vorgang unter gewissen pathologischen Verhältnissen Platz greift, wird nicht allerseits von den betreffenden Autoren bestätigt.

Um die Frage zu beleuchten, in welchem Umfange das Keratohyalin und das Eleidin bei einem durch pathologische Verhältnisse hervorgerufenen Verhornungsprozesse sich betei- 
ligt und wie sich diese beiden Substanzen an dem Aufbaue der Hornlamellen beteiligen, hat Bizzozero an den Sohlenballen von Kaninchen und auch an menschlicher Haut bei verschieden erzeugter Entzündung die einzelnen Erscheinungen studiert und konnte verschiedene Vorgänge verzeichnen:

„Solange die Entzündung florid ist, wird die Bildung von Keratohyalin und Eleidin aufgehoben. Bei der Regeneration der Hornschicht nach diesen Prozessen entsteht immer zuerst Keratohyalin, dann Eleidin und dann normale Hornschicht. Das Aultreten von färbbaren Kernen in der Hornschicht erfolgt bei pathologischen Prozessen immer nach Sistierung der Bildung von Keratohyalin und Eleidin (Parakeratose). Die Parakeratosen sind kaum wesentlich durch Leukozytenauswanderung bedingt (Kromayer), da dieselbe sehr oft fehlte."

Nach Durchlesen der Arbeit Bizzozeros hat es mich interessiert, wie sich wohl diese zwei Substanzen bei unserem syphilitischen Prozesse verhalten, nämlich, ob sie überhaupt vorhanden und wenn, wie sie verteilt waren, und ob man auch bei einer chronischen Entzündung beiläufig ähnliche Erscheinungen finden würde, wie sie Bizzozero bei akuten Entzündungsformen konstatierte; weiter, ob man aus dem gegenseitigen Verhalten beider Substanzen auf die einzelnen Stadien des Entzündungsprozesses irgendwelche Schlüsse ziehen darf und endlich, ob und in welcher Art und Umfange sich an dem Aufbaue der Hornschichte beide Substanzen beteiligten.

$\mathrm{Zu}$ diesem $\mathrm{Z}$ wecke wurden Kohlensäure-Gefrierschnitte nach Angabe von Bizzozero angefertigt und diese nach Buzzi mit Kongorot gefärbt und mit Hämalaun nachgefärbt.

Wir fanden bei dieser Färbemethode, daß trotz des anbaltenden Entzündungsprozesses die Bildung von Keratohyalin und Eleidin nicht aufgehoben war und sahen, daß das Keratohyalin und die vorhandenen Kerne sich blau färben und zwar in den 5-7 obersten Reihen des Stratum granulosum. Iu der obersten Reihe, wo die Konturen einzelner Zellen nicht mehr sehr scharf hervortreten, fanden wir sowohl in dem Innern der Zellen, als auch in ihrer nächsten Umgebung und dann dort, wo normalerweise das Stratum Iucidum beginnt, stellenweise kleinere Gruppen, oder stellenweise längere, zusammenhängende, 
sehr schmale Massen von orangerot gefärbten feinen Körnern, die wir als Eleidin auffaßten.

Ein Auftreten von färbbaren Kernen in der ganzen Hornscbicht war nirgends zu sehen und trotzdem auch hie und da ausgewanderte Leukozyten in der Epithelschicht konstatiert werden, fanden wir die Hornschicht nirgends durch eine neugebildete parakeratotische Schicht unterbrochen. Die Hornschicht schneidet gegen die Malpighische scharf ab; Übergänge von der cinen Schichte in die andere existieren nicht.

Wir müssen daher annehmen, dab sich andere Bildungsformen und -Fasen einstellen, wenn pathologische Verhältnisse von außen auf das Stratum corneum einwirken und von da aus gegen die tieferen Schichten weitergeleitet werden als in jenen Fällen, bei welchen sich ein pathologischer Prozeß, wie es bei den sekundären Hyperkeratosen der Fall ist, in der tiefen Subkutis abspielt und sich nur in den obersten Hautschichten abspiegelt; darum finden wir wahrscheinlich keine Mitosen, keine Parakeratose, sondern nur eine abnorm breite Körnerschichte und eine schmale Zone von Eleidinkörnchen; daran schließt sich scharf die ungewöhnlich mächtige, einheitliche Hornmassenlage an.

Es sei uns also die Annahme gestattet, daß, da in unsern Präparaten unzweifelhafte entzündliche Erscheinungen in der Kutis nachgewiesen wurden, das Rete Malpighii schnell und prompt auf diese !kutane Entzündung mit Proliferation reagierte und zur Hornmassenbildung AnlaB gegeben hat. Wie aber diese Auflagerungen im Stratum corneum zustande gekommen sind, das erklären uns die Befunde in unseren Schnitten nicht mehr, da wir ja bereits ausgebildete Effloreszenzen untersuchten, die nicht mehr die beginnende Verhornung zeigen können. Auch das Stück, welches sechs Tage nach der Injektion exzidiert wurde, zeigte keine Anzeichen, aus welchen man auf eine ursprüngliche Entstehung aus der verkehrten Reihenfolge schließen könnte.

Die nach der Ernstschen Methode (Gramfärbung) gefärbten Schnitte ergaben uns über den Modus der pathologischen Verhornung in diesem Falle keinen besonderen Aufschluß. 
Selbstredend reichen die bisherigen Forschungen in dieser Richtung nicht so weit aus, um diesbezüglich ein endgültiges Urteil fällen zu können und es wäre daher von großem Nutzen, wenn sich die Möglichkeit öfters darbieten würde, insbesondere chronische, zur Verhornung führende Prozesse in ihren verschiedenen Entwicklungsphasen eingehend beobachten und beurteilen zu können.

Ob nun bei der Ätiologie und Pathogenese der Hyperkeratosen ein infektiöser Ursprung ( $U \cap n \cap$ ) im Spiele ist, bleibt fraglich.

Tomaseli meint, es handle sich um Autotoxine, um keratophore Toxine, die aus Ernährungsstörungen hervorgegangen sind.

Jarisch dagegen glaubt, daß es sich bei den einzelnen Hyperkeratosen nicht nur um quantitative, sondern auch um qualitative Unterschiede in der Verhornung und Zusammensetzung der Hornzellen handeln könne. Wenn wir in dieser Richtung unsern luetischen Prozeß betrachten, so sehen wir, daß er durch seine reine Hyperkeratose der Schwiele am nächsten steht; wir finden aber in unsern Schnitten kein Stratum lucidum, welches bei der Schwiele noch deutlicher hervortritt, als dies gewöhnlich in der normalen Fußsohlenhaut zu sein pflegt; ebenso finden wir keine Verschmälerung des Rete Malpighii, die normalerweise bei der Schwiele vorkommt.

Von einem Klavus zu sprechen, ist noch weniger begründet, denn bei diesem ist die Hyperkeratose in der Mitte des betreffenden Hautgebildes besonders hochgradig entwickelt und zwar in der Weise, da $B$ die Hormmassen in Gestalt eines konischen Zapfens in das Rete hineinragen. Infolge des Druckes dieses Hornkegels wird das Rete Malpighii atrophisch und wir finden es im mikroskopischen Präparate verschmälert.

Solche Bilder, wie wir eben geschildert, sind uns mikroskopisch nicht zu Gesicht gekommen, es hat also weder die eine noch die andere Benennung ("Tyloma syph.", "Clavus syph.") ihre Berechtigung.

Aus diesen Ausführungen ergibt sich, daß manchmal im Verlaufe einer syphilitischen Allgemeinerkrankung bei einzelnen 
Individuen Hyperkeratosen an den Handflächen und Fußsohlen sich ausbilden können.

Ob die Ursache in der Eigentümlichkeit des Krankheitsprozesses, in der Giftintensität des Syphiliserregers, in der allgemeinen Beschaffenheit des Kranken oder in der lokalen minderen Widerstandsfähigkeit $\mathrm{zu}$ suchen ist, läßt sich heute auf Grund der bisher vorgenommenen Forschungen und der darüber vorliegenden Resultate nicht bestimmt beantworten; es dürften dabei wahrscheinlich immer mehrere Auslösungsarten im Spiele sein.

Das bei dem ron uns erwähnten Proze $B$ als alleinige Ursache lokale, mechanische oder chemische länger andauernde Reize, wie sie Lewin, Brooke, Verchère bei einzelnen anderen Hyperkeratosenformen der Handteller und Fußsohlen verzeichnet haben, angesehen werden könnten, ist mit Rïcksicht auf das früher angeführte nicht wahrscheinlich; viel eher ist man gezwungen anzunehmen, daß solche Reizmomente zwar zur Ausbildung der "Clavi syphilitici“ gewissermaßen beitragen, nicht aber die alleinige auslösende Ursache abgeben können.

Wohl könnte man zu dem vorbesprochenen ersten und zweiten Fall bemerken, daß die "Clavi syphilitici" bei ihnen als ein Folgezustand, entweder der Hände harter Arbeit, oder der militärischen Übungen, anzusehen sind, aber in Anbetracht eines vor Jahren sich ergebenen analogen Falles, der nachstehend geschildert wird, duirfte man zu einem anderen Schlusse gelangen. Ich bekam nämlich einen Kranken, der zuvor infolge Rheumatismus eine 8wöchige Badekur in Teplitz-Schönau durchmachte. Trotzdem er seines Zustandes wegen nahezu fort an las Bett gebunden war, hatten sich dennoch während seines Aufenthaltes in dem erwähnten Kurorte zahlreiche Schwielen an seinen Fulsohlen und Handtellern entwickelt und erst als sie als „Clavi syphilitici ${ }^{\alpha}$ erkannt und dann entsprechend antiluetisch (Schmierkur) behandelt wurden, schwand der Rheumatismus und wach diesem auch die Klavi, wiewohl der Kranke während dieser Behandlung verhältnismäßig viel herumging. Auch im jetzigen dritten Falle kann man dem Entstehen der Klavi keine mechanische Ursache beimessen. Mithin wäre 
man zu dem Schlusse berechtigt, daß bei diesen Kranken nur die in der Tiefe begonnene spezifische Entzündug die Hyperkeratosen bervorgerufen hat.

Die Behandlung meiner in der Einleitung erwähnten vier Fälle nahm eine bedeutend längere Zeit in Auspruch als in jenen drei Fällen, wo Salvarsan angewendet wurde, obwohl der Regenerationsproze $\beta$ in allen Fällen den gleichen Charakter besaß. Hiebei muß hervorgehoben werden, daß bei der $\mathrm{Hg}$-Kur dle Einzelerscheinungen der lokalen Veränderungen, welche das Fortscbreiten des Heilungsprozesses andeuten, nicht so ins Auge fallen, da sie nur sehr langsam vor sich gehen, während bei der Heilmethode mit Salvarsan die erwähnten Veränderungen in rascher Folge sich einstellen, ohne daß irgendwelche Lokalapplikation (Erweichungsprozeduren) angewendet worden wäre.

Bei der Behandlung der früheren Patienten dagegen wurde immer ein großer Wert auf die lokale Anwendung von Sublimatbädern, Guttaplast Hydrargyrum Nr. 15 Beiersdorf, Salizylseifenpflaster $20 \%$ und Dunstumschläge gelegt; doch 5 bis 7 Wochen waren verstrichen, ehe die Haut der Hand- und Fußflächen ihr normales Aussehen erlangte.

In welcher Form das $\mathrm{Hg}$ bei solchen Fällen angewendet wird, dürte auf den Verlauf kaum von Einfluß sein; auch eine kombinierte Hg- und Jodkalibehandlung (wie ich sie in einem früheren Falle anwendete) beeinflußte nicht intensiver die hyperkeratotischen luetischen Erscheinungen und beschleunigte nicht deren Abheilung. Vielmehr ging dieselbe in dem Tempo vor sich, wie bei den Effloreszenzen an den übrigen Körperstellen und Schleimbäuten; ja in der Regel iiberdauerten die Hyperkeratosen die anderen Lueserscheinungen.

Von den drei mit 603 behandelten Fällen sind bei dem Rekruten die Hornmassen und Infiltrate nach 18 Tagen, bei dem zweiten Falle nach 8 Tagen, bei dem dritten nach 21 Tagen gänzlich geschwunden, was gewiß nur der Einwirkung des neuen Mittels zugeschrieben werden muß. Bemerkenswert ist noch weiter, daß bei dem Rekruten 4 Wochen nach der Injektion mit 606 sich eine Rezidive in Form von nässenden Papeln im Nabeltrichter und in Form eines tiefen Geschwüres (Spiro- 
chaetenbefund einigemal positiv) an der linken Tonsille in der Narbenstelle nach dem ersten Geschwür einstellte. Auch die Wassermannsche Reaktion, welche 3 Wochen nach der Arsenobenzolinjektion negativ geworden ist, zeigte nach Ausbruch der neuen Rezidive einen Umschlag wieder ins Positive. Nachdem die Rezidive neuerdings an denselben Stellen, wie bei der ersten Erkrankung, aufgetreten ist (Nabeltrichter, linke Tonsille), zwang uns das Interesse durch einen Versuch festzustellen, ob durch erneuerte mechanische Ursachen (forciertes Gehen und Arbeiten) auch an den Handtellern und Fußsohlen die Hyporkeratosen abermals hervorgerufen werden könnten; aber dieser Versuch fiel vollkommen negativ aus. Im Laufe von 3 Monaten wurde derselbe Kranke wiederholt untersucht Obwohl er bei der Kompagnie die ganze Ausbildungsperiode durchgemacht hat, wurden bei $\mathrm{ihm}$ an den Handflächen und Fußsohlen keine Veränderungen nicht einmal in Form normaler Schwielen an den Prädilektionsstellen vorgefunden.

Zum Schlusse erlaube ich mir die Resultate dieser Arbeit im folgenden Sinne zusammenzufassen.

Der luetische Prozeß kann entweder durch im Blute zirkulierende toxische Substanzen oder durch Spirochaeten selbst eingeleitet werden. In der Kutis und Subkutis sieht man bei diesem Prozesse, daß sämtliche Gefäßschlingen eine mächtige Anhäufung eines kleinzelligen Infiltrats zeigen, welches mit den feinsten Gefäßverästelungen bis in die Spitzen der Papillen, ja sogar in den basalen Teil des Stratum Malpighii eindringt. Im Verlaufe des spezifischen Prozesses werden diese Schichten gereizt und es kommt zur gesteigerten Epithelzellproliferation. Letztere schreitet im viel rascheren Tempo als im normalen Zustande fort, weswegen sich die Zellschichten aneinander anbäufen. Hiebei wandelt sich ein größerer Teil der Zellen in den obersten Reihen des Stratum granulosum durch stete Apposition und festes Zusammenhalten in kernlose Hornlamellen um.

Die Abnützung und der Austausch der Hornschichte kann nicht gleichmäßig mit der gesteigerten Vermebrung der 
Zellen ror sich gehen; auch findet dabei die Austrocknung und Entfettung der Hornschichte nicht im ausgiebigen Maße statt, folglich kommt es zur Ausbildung von lokalen Hyperkeratosen.

Aus dem Vorgesagten geht hervor, daß die spezifische luetische Entzündung in der Kutis und Subkutis allein Hyperkeratosen der Hohlhand und FuBsohle hervorzurufen vermag.

Zum weiteren Ausbaue einer mächtigen Hornschichtauflagerung kann eventuell auch noch der Druck der bereits geschweißten Hornmassen beitragen, respektive spielen dabei auch noch andere äußere Einflüsse eine gewisse Rolle mit.

Meinem hochgeschätzten Lehrer P. T. Prof. Janorský, von dem die Anregung zu dieser Arbeit an mich ausging, erlaube ich mir hiefür sowie für die mir zur Verfügung gestellte Literatur meinen tiefgefühltesten Dank darzubringen.

\section{Literatur.}

Bering. Zur Kenntnis der Hyperkeratosen. Archiv f. Derm, und Syph. Bd. LXXVI. 1905.

Besnier. Keratodermie symmetrique des extrémités. Atlas intern. des maladies rares de la peau. 1889.

Bizzozero. Über die Dariersche Dermatose. Arch. f. Derm. u. Sypb, Bd. XCIII. 1908.

- Experimentelle Studien über Keratohyalin u. Eleidin n. Parakeratose. Arch. f. Derm. u. Syph. Bd. XCVII.

B laschko. Über den Verbornungsprozeß mit Demonstrationen von Präparaten. Verhandl. der deutschen dermat. Gesellsch. I. Kongreß. Prag 1889.

Brooke. Bemerkungen über einige Keratosen der Handteller und Fußsohlen. Monatsh. f. prakt. Dermatol. Bd. XII. 1. H. 1891.

$\mathrm{Buk}$ ov ský. Psorospermosis follicularis vegetans Darier. Arch. $f$. Derm. u. Syph. Bd. LXXV. 1905.

Buschke. Über Exantheme bei Gonorrhoe. Arch. f. Derm. u. Syph. Bd. XLVIII. 1899. 
Dreysel und 0 ppler. Beiträge zur Kenntnis des Eleidins in normaler und pathologisch veränderter Haut. Arch. f. Derm. u. Syph. Bd. XXX. 1895.

Dubreuilh. Artikel in der Pratique dermatologique.

During, von. Hyperkeratosen. Die Deutsche Klinik. 14. Vorlesung. Leyden-Klemperer 1905.

Ehrmann. Kompendium der spez. Histopathologie der Haut. 1906.

Ernst. Studien über die normale Verhornung mittelst der Grammschen Methode. Arch. f. mikroskop. Anat. 1896. Bd. XLVII.

Fabry. Über einen Fall von Hyper- and Parakeratosis acquisita corporis. Arch. f. Derm. u. Syph. Bd. LXV.

Fischel. Zur Behandlung der Psoriasis palm. et plant. Prager med. Wochenschr. 1905. Nr. 2.

Jada 8 ohn. Keratosen. Handbuch der prakt. Medizin. EbsteinSchwalbe 1901 .

J a novský. Hyperkeratosen. Mračeks Handbuch der Hautkrankkeiten. Bd. III. 1904 .

Heft IV. 1890.

- Acanthosis nigricans. Intern. Atlas seltener Hautkrankheiten.

Janošík. Histologie und mikroskop. Anatomie. (Böhmisch.) 1892.

Jarisch. Die Hautkrankheiten. 1900, 1908.

Syph. 1895.

- Zur Kenntnis der Darierschen Krankheit. Arch. f. Derm. u.

Jessner. Kompendium der Hautkrankheiten. 1904.

Kreibich. Lehrbuch der Hautkrankheiten. 1904.

Bd. LXXX. 1906 .

Kromayer. Allgemeine Dermatologie. 1896.

Kaposi. Patbologie und Therapie der Hautkrankheiten. 1899.

La ̌ Gesellsch. I. KongreB Prag 1889.

Lesser. Lehrbuch der Haut- und Geschlechtskrankheiten. 11. Aufl. Lewin. Clavi syphïitici. Arch. f. Derm. u. Syph. 1893.

Merk. Über den Bau der menschlichen Hornzelle. Arch. f. mikroskop. Anatomie. Bd. I.VI. 1900.

Mraček. Syphilis der Haut, Mračeks Handbuch der Hautkrankheiten. Bd. IV.

Mourek. Ein Beitrag zur Lebre von Dermatosis Darier. Arch. $f$. Derm. u. Syph. 1894.

Pöhlmann. Dariersche Erkrankung in drei Generationen. Arch. f. Derm. u. Syph. Bd. LCVII, 1909.

Pollitzer. Acanthosis nigricans. Intern. Atlas seltener Hautkrankheiten. Heft IV. 1890.

Rab 1. Untersuchungen über die menschliche Oberhaut und ihre Anhangsgebilde mit besonderer Rücksicht auf die Verhornung. Arch. f. mikroskop. Anatomie. Bd. XLVIII. 1897.

Secchi. Zur Topographie des elastischen Gewebes der normalen menschlichen Haut. Arch. f. Derm. u. Syph. Bd. XXXIV. 1896.

Schütz. Über die vom Druck unabhängige Tylosis palmarum der Erwachsenen. Arch. f. Derm. u. Syph. Bd. LIX. 1900.

$S k l a r e k$. Beiträge zur Kenntnis der Schwielen und Hühneraugen Arch. f. Derm. u. Syph. Bd. LXXXV. 1907.

Spietschka. Über Dystrophie papillaire et pigmentaire (Akanthosis nigricans). Arch. f. Derm. u. Syph. Bd. XLIV. 1898.

Sa m berger. Zur Pathologie der Hyperkeratosen. Keratoma plantare hereditarium. Arch. f. Derm. u. Syph. Bd. LXXVI, LXXVII. 1905. 
Unna. Über das Keratoma palmare et plantare hereditarium. Arch. f. Derm. u. Syph. 1883.

- Über das Wesen der normalen und pathologischen Verhornung. Monatsbefte f. prakt. Derm. Bd. XXIV. 1897.

- Histopathologie der Hautkrankheiten. 1896.

Verchère. Akutes Karbolekzem des Handtellers, nachfolgende trophische Störungen. France méd. Juli 1894. Ref. in Monatshefte f, prakt. Dermat. Bd. XX. 1895. I. p. 465.

Vörner. Zur Keuntnis der Keratoma hereditarium palmare et plantare. Arch. f. Derm. u. Syph. Bd. LVI. 1901.

Waelsch. Zur Histologie der Arsenkeratose. Arch. f. Derm. u. Syph. Bd. LXXXVI. 1907.

Weidenreich. Über Bau und Verhornung der menschlichen Oberhaut. Arch. f. mikroskop. Anat. Bd. LVI. 1900.

\section{Erklärung der Abbildungen auf Taf. V.}

Erstes Bild: Stratum corneum vor der Injektion.

Zweites Bild: Stratum granulosum mit Keratohyalin und Eleidin. Drittes Bild: Entzündung in der Kutis und Subkutis vor der In. jektion mit Salvarsan.

Viertes Bild: Stratum corneum und Entzündung 7 Tage nach der Injektion. 

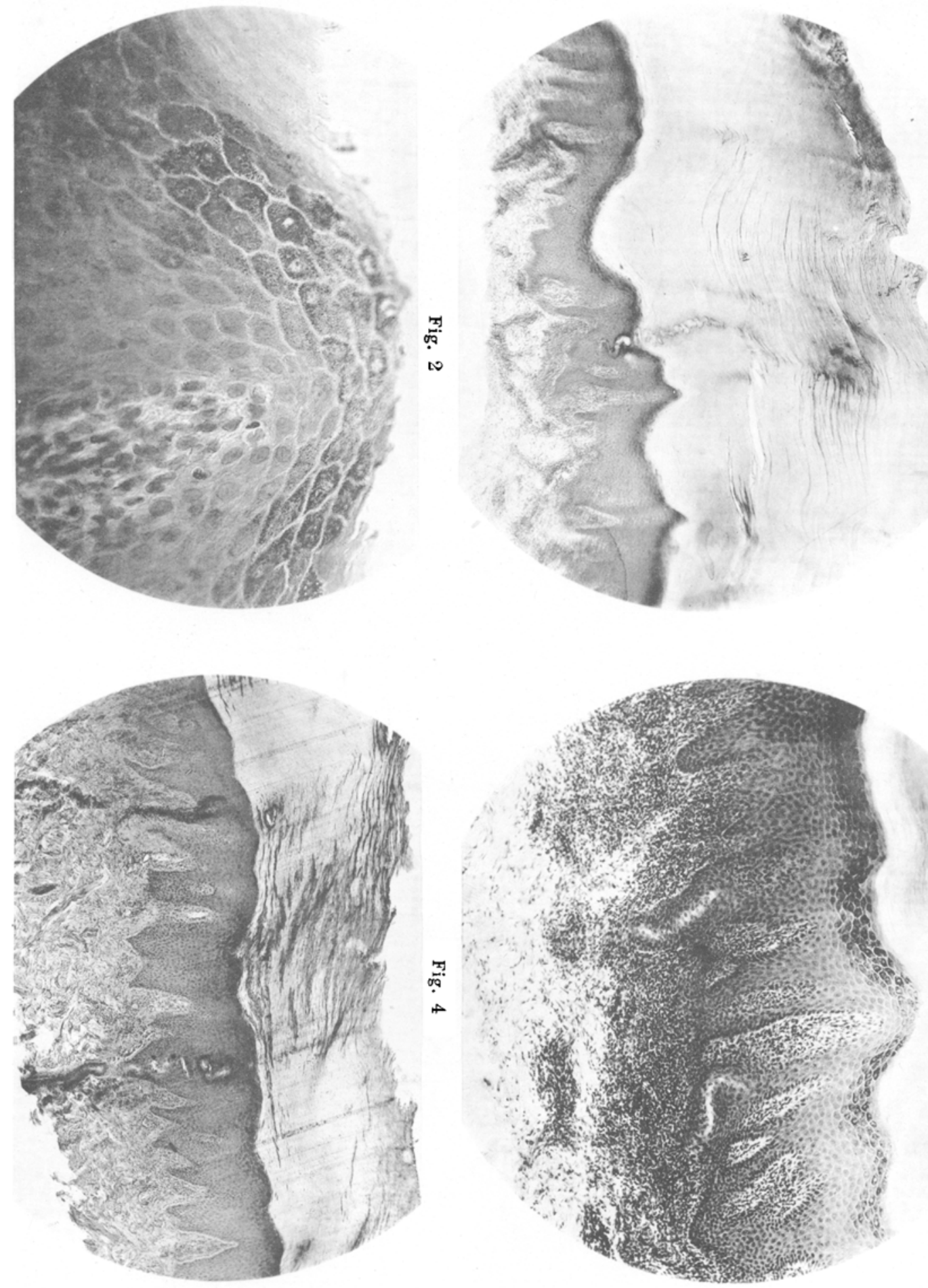